\title{
Surgical Management of Small Bowel Neuroendocrine Tumour Symposia
}

\author{
Jonathan Koea ${ }^{1} \cdot$ Janice Pasieka $^{2}$ on behalf of The Commonwealth Neuroendocrine \\ Tumour Research Collaborative (CommNETs) Surgical Section
}

Published online: 29 June 2020

(C) Société Internationale de Chirurgie 2020

\section{Introduction}

While rare, the incidence of small bowel neuroendocrine tumours (SBNETs) is increasing and they are now the most frequently encountered small bowel tumour [1]. Up to one quarter of SBNETs present acutely, many present as an incidentally, and most present with metastatic stage IV disease [2]. Neuroendocrine tumours usually require consultative, multidisciplinary management at specialized centres. Prolonged survival with good quality of life can be achieved in patients even with extensive metastatic disease. However, with the rising incidence and the presentation of complications from advanced disease such as obstruction, ischemia, perforation and bleeding, many of the patients with SBNET will be encountered by every general and subspecialty surgeon undertaking acute and emergency surgery. The dilemmas that are faced by the operating surgeon who encounters this disease include: 1 . How to manage of the SBNET presenting with acute complications such as obstruction and perforation? 2. The extent and technique of regional lymphadenectomy, how to do it safely? 3. What are the significance and the management of multifocal disease? 4. What is the recommended management of the primary and regional disease in patients with distant

This article is contribution to the Symposium entitled, "Surgical Dilemmas and Challenges for the Operating Surgeon in the Management of Small Bowel Neuroendocrine Tumours: A CommNETs Symposium".

Jonathan Koea

jonathan.koea@waitematadhb.govt.nz

1 The Department of Surgery, North Shore Hospital, Private Bag 93503, Takapuna, Auckland 0620, New Zealand

2 Tom Baker Cancer Centre, Calgary, Alberta, Canada (predominately liver) metastatic disease? 5. Finally, what is the best palliation and management for the rare patients with unresectable SBNET? The following symposium attempts to answer and address these practical issues faced by the operation surgeon in patients with SBNETs. In this issue of the World Journal of Surgery, five surgeons from Canada and New Zealand present a symposia of concise reviews summarizing the management options for patients presenting with complex SBNET disease.

Based on New Zealand data, the incidence of NETs is 8/100,000 with 75 gastrointestinal NETs presenting annually, in Australia 350 patients are diagnosed annually and in Canada up to 1800 patients are diagnosed annually. Since all three countries share similar health systems and have availability of biobanking and metabolic imaging, the Commonwealth Neuroendocrine Tumour research collaborative (CommNETs) was established in 2015. The five surgical authors represent the surgical section of the CommNETs which operates within the Canadian Cancer Trials Group and the Australasian Gastrointestinal Trials Group [3]. CommNETs has a multidisciplinary representation and includes all clinical specialties involved in the care of NET patients as well as patient representatives and members of the Unicorn Foundation of New Zealand, CNETs from Canada and NeuroEndocrine Cancer from Australia. The challenges faced by surgeons treating NET patients, as outlined in this symposia, are universal. We hope that with the collective symposium can contribute to the global initiatives to improve the care and outcomes for patients with NETs worldwide.

\section{Compliance with ethical standards}

Conflict of interest The CommNETS Collaboration is supported by unconditional educational grant from Ipsen Canada and an unconditional sponsorship grant from Ipsen Australia. 


\section{References}

1. Bilimoria KY, Bentrem DJ, Wayne JD et al (2009) Small bowel cancer in the United States: changes in epidemiology, treatment and survival over the last 20 years. Ann Surg 249:63-71

2. Manguso N, Gangi A, Nissen N et al (2018) Long-term outcomes after elective versus emergency surgery for small bowel endocrine tumors. Am Surg 84:1570-1574
3. https://gicancer.org.au/health/-professional/commnets/. Accessed 31 May 2020

Publisher's Note Springer Nature remains neutral with regard to jurisdictional claims in published maps and institutional affiliations. 